\title{
La question de la progressivité des apprentissages en production écrite à l'école élémentaire française : le cas de la segmentation en phrases
}

\author{
Paolacci, Véronique ${ }^{1} \&$ Rossi-Gensane, Nathalie ${ }^{2}$ \\ ${ }^{1}$ CLLE ERSS (UMR 5263), Université Toulouse-Jean Jaurès \& ESPE \\ ${ }^{2}$ ICAR (UMR 5191), Université Lumière Lyon 2 \\ veronique.paolacci@univ-tlse2.fr, nathalie.rossi-gensane@univ-lyon2.fr
}

\begin{abstract}
Résumé. D'une manière générale, les apprentissages effectifs des élèves sont difficiles à apprécier. Nous nous interrogeons dans cet article sur la progressivité des apprentissages concernant le découpage en phrases dans la situation de production écrite à l'école. Comment viser une cartographie de ces apprentissages rédactionnels ? Quels sont les indicateurs à identifier afin d'aider les élèves à améliorer leur écrit ? En réponse à ces questions, nous proposons une grille de critères de progressivité pour aborder la question du découpage en phrases d'un texte d'élève et ainsi interroger les emplois des signes de ponctuation et des connecteurs. Notre analyse se fonde sur des textes d'élèves de CM2 qui font partie d'une lecture accompagnée d'écrits fréquents et qui, collectés à quatre mois d'intervalle, répondent à une même consigne d'écriture. Dans le cadre de l'article, quatre cas contrastés sont caractérisés à partir des critères de la grille présentée. Nous concluons sur l'hétérogénéité des performances des élèves et sur la nécessité d'enseigner explicitement la segmentation en phrases dans un travail étayé de réécriture.
\end{abstract}

\begin{abstract}
In a general way, pupils' effective learning is quite difficult to pinpoint. In this article, gradualism in learning is questioned as regards sentence segmentation in the production of texts at the end of French primary school. We wonder how writing apprenticeship in this particular field can be accounted for, so as to help pupils improve their writing skills. We therefore establish a grid of criteria bearing on gradualism with sentence segmentation, with special attention to the use of punctuation marks and connectors. Our analysis relies on two sets of texts following the same instructions which have been collected at an interval of four months. Four contrasted cases are then characterized on the basis of the established criteria. We conclude on pupils' heterogeneous writing skills and recommend that sentence segmentation should be explicitly taught.
\end{abstract}




\title{
Introduction $^{1}$
}

Nombreux sont les enseignants qui, soucieux de répondre aux difficultés de leurs élèves, s'interrogent sur la progressivité des apprentissages. Ce concept de «progressivité » peut être rapproché d'autres concepts didactiques comme ceux de «progression» et de «programmation». Toutefois, si la progression et la programmation concernent les objets de savoirs et le travail de l'enseignant quant à la planification des enseignements (Nonnon, 2010), la progressivité des apprentissages se situe du côté de l'élève et est déterminée par le rapport aux savoirs de ce dernier ainsi que par les obstacles qu'il doit franchir pour acquérir ces savoirs. Progression, programmation et progressivité sont intimement liées comme le précise Nonnon $(2010: 7)$ :

\begin{abstract}
Concevoir et gérer une progression implique l'anticipation mais aussi l'ajustement en fonction d'une analyse de ce que font les élèves, de ce qu'ils apprennent ou n'apprennent pas. [...] Dans cette perspective, concevoir, mettre en œuvre, réguler une organisation temporelle des enseignements et une progressivité des apprentissages peut être appréhendé dans une perspective d'analyse du travail comme une des composantes les plus difficiles et décisives de l'activité enseignante. Il faut la replacer dans les conditions effectives de l'exercice du métier, en tenant compte des tensions qu'induisent les écarts entre les objectifs programmés et les apprentissages effectifs des élèves, entre les rythmes d'apprentissages des élèves dans le cadre d'un enseignement collectif.
\end{abstract}

La progressivité des apprentissages, comme la progression et la programmation, place au cœur de la réflexion didactique le temps nécessaire pour apprendre effectivement (Nonnon, 2010). À la réflexion de l'enseignant sont ajoutés la différenciation des savoirs et le rythme d'acquisition des apprenants, comme le souligne ci-dessus Nonnon (2010). Penser la progressivité des apprentissages est par conséquent, pour le professeur, une tâche complexe, particulièrement pour les apprentissages au niveau de l'écrit.

Aussi, notre étude a pour objectif d'apporter une pierre à l'édifice de cette réflexion didactique pour ce qui touche à un aspect particulier (parmi de nombreux autres) de la production écrite (certes multidimensionnelle) : la question de la segmentation du texte. Ce travail entend ainsi contribuer à la mise en œuvre d'une cartographie des compétences rédactionnelles.

Plusieurs questions fédèrent notre réflexion: quels sont les indicateurs de progrès qui permettent d'approcher la progressivité des apprentissages concernant la segmentation en phrases d'un texte? Comment la compétence démarcative chez un même élève évolue-t-elle au cours du temps ? Et, d'ailleurs, y a-t-il toujours évolution? Nous posons comme hypothèse que cette compétence s'inscrit dans une dynamique que nous nommerons « dynamique démarcative».

Nous reviendrons, dans un premier temps, sur la difficulté de circonscrire la notion de phrase aux niveaux linguistique et didactique. Nous préciserons, dans un deuxième temps, nos choix méthodologiques et exposerons, dans un troisième temps, les résultats de notre étude sous la forme de portraits d'élèves, avant de conclure sur des propositions didactiques. 


\section{La phrase : une notion difficile à enseigner}

La phrase est une notion difficile à appréhender, car multiforme, sur un plan linguistique (1.1) et sur un plan didactique (1.2).

\subsection{La phrase : une notion linguistique difficile à circonscrire}

Comme il a été souvent souligné (voir notamment Berrendonner, 2002, et Groupe de Fribourg, 2012), les critères définitoires traditionnels de la phrase sont de divers ordres : typographique (la phrase commence par une majuscule et finit par un signe de ponctuation forte), prosodique (prosodie et ponctuation étant censées se correspondre, tout au moins en partie), sémantique (la phrase est dotée d'un sens, parfois qualifié de «complet »), syntaxique (la phrase est constituée de l'ensemble des éléments reliés par des rapports de dépendance à un même élément central) ou plus particulièrement catégoriel (lorsque l'élément central est ramené à un verbe), illocutoire (la phrase est associée à une modalité énonciative - déclarative, interrogative ou injonctive). Ces critères hétérogènes sont parfois contradictoires et, dans les faits, le critère typographique prévaut.

\subsection{La phrase : une notion enseignée surtout en réception}

Quels critères afférents à la notion de phrase peut-on observer dans les textes officiels et dans les supports didactiques ? Quels sont les « repères de progressivité $»^{2}$ concernant la notion de phrase ? Observe-t-on un épaississement de cette notion par l'ajout de « facettes $»^{3}$ ?

\subsubsection{La phrase dans les textes officiels}

Dans les programmes de 2008, encore en vigueur au moment où nous écrivons ces lignes, la phrase est avant tout présentée dans une perspective de réception, dans le domaine de la «lecture », comme il pouvait être attendu, mais aussi dans le domaine de la «grammaire ». Dans le premier domaine est explicitement visée en CE2, CM1 et CM2, de manière générale, la «compréhension des phrases » (B.O. 2008: 21). Dans le second domaine sont associés à cette perspective de réception les critères typographique (en particulier en $\mathrm{CP}$ ) et catégoriel (aux cinq niveaux de l'école élémentaire). Ainsi, en CP et CE1, «La première étude de la grammaire concerne la phrase simple. Les marques de ponctuation et leur usage sont repérés et étudiés. Les élèves apprennent à identifier la phrase [...]. Ils apprennent à repérer le verbe d'une phrase et son sujet» (B.O. 2008 : 17). Selon les progressions pour le CP (B.O. 2008: 32), il s'agit d'«identifier les phrases d'un texte en s'appuyant sur la ponctuation (point et majuscule) ». Les progressions pour le CE1, pour le CE2 et pour le CM1 préconisent respectivement d' « identifier le verbe et son sujet » dans « la phrase simple où l'ordre syntaxique régulier sujet-verbe est respecté » (B.O. 2008: 32), d'«identifier le verbe conjugué dans une phrase simple et fournir son 
infinitif» (B.O. 2008 : 36) et d' « identifier les verbes conjugués dans des phrases complexes et fournir leurs infinitifs » (B.O. 2008 : 36). On notera par ailleurs que le critère typographique est convoqué sous forme de sous-entendu alors que le critère catégoriel établissant une relation bi-univoque entre phrase (simple) et verbe conjugué est présent en tant que présupposé.

Comme il pouvait également être attendu, la phrase est présentée dans une perspective de production dans le domaine de l'«écriture »: en $\mathrm{CP}$ et $\mathrm{CE} 1$, « les élèves apprennent à rédiger de manière autonome un texte court », ce qui inclut «construire et enchaîner des phrases » (B.O. $2008: 17$ ). Les progressions dans ce domaine précisent, pour le CP, «écrire sans erreur, sous la dictée, des syllabes, des mots et de courtes phrases dont les graphies ont été étudiées », « concevoir et écrire collectivement avec l'aide du maître une phrase simple cohérente, puis plusieurs » et, pour le CE1, «écrire sans erreur, sous la dictée, des phrases ou un court texte (5 lignes), préparés ou non, en utilisant ses connaissances orthographiques et grammaticales » (cette dernière préconisation est répétée à l'identique dans la page suivante, cette fois dans le domaine de l'«orthographe »), «concevoir et écrire de manière autonome une phrase simple cohérente, puis plusieurs, puis un texte narratif ou explicatif de 5 à 10 lignes » (B.O. 2008 : 31). Dans le domaine de l'«orthographe », le critère typographique est mis à l'honneur dans cette perspective de production par les progressions pour le $\mathrm{CP}$ : « commencer à utiliser correctement la majuscule (débuts de phrase [...]) » (B.O. 2008 : 32). Dans le domaine de la « grammaire », la perspective de production est associée, à partir du CE1, au critère illocutoire (même si, en premier lieu, les deux perspectives paraissent équilibrées, le programme du CE2, du CM1 et du CM2 mentionnant tout d'abord à la fois « [1a] connaissance et [l'] emploi pertinent des phrases déclarative, interrogative, injonctive et exclamative ${ }^{4}$ » (B.O. 2008 : 22)). Ainsi, les progressions, tout en mêlant polarité (ou forme de phrase) et modalité énonciative (ou type de phrase), précisent «approche des formes et types de phrase : savoir transposer oralement une phrase affirmative en phrase négative ou interrogative » pour le CE1 (B.O. 2008: 32), « transformer une phrase simple affirmative en phrase négative ou interrogative, ou inversement » pour le CE2 (B.O. $2008: 36$ ), « construire correctement des phrases négatives, interrogatives, injonctives » pour le CM1 (B.O. 2008 : 36) et «construire correctement des phrases exclamatives » pour le CM2 (B.O. $2008: 36)$.

Pour ce qui est, plus particulièrement, de ladite phrase complexe, on note la même tendance. La perspective de réception prévaut dans le domaine de la grammaire. Est ainsi visée en CM2 la «distinction entre phrase simple et phrase complexe; entre proposition indépendante (coordonnée, juxtaposée), proposition principale et proposition subordonnée» (B.O. 2008: 22). Les progressions pour le CM2 précisent: «comprendre la distinction entre phrase simple et phrase complexe », « reconnaître des propositions indépendantes coordonnées, juxtaposées », « reconnaître la proposition relative (seulement la relative complément de nom)» (B.O. 2008: 36). On peut toutefois remarquer que les progressions pour le CE2 prennent en compte le fait de « relier des phrases simples par des mots de liaison temporelle (ex. les adverbes puis, alors...) »(B.O. 2008: 36). En revanche, la perspective de production est représentée dans le domaine de la «rédaction », comme il apparaît dans les progressions pour le CM1 : « savoir amplifier une phrase simple par l'ajout d'éléments coordonnés ( $e t, n i, o u$, mais entre des mots ou des phrases simples; car, donc entre des phrases simples) [...]» (B.O. $2008: 35$ ).

\subsubsection{La phrase dans les manuels}

Les manuels Pépites, récemment publiés chez Magnard, sont déclinés à tous les niveaux de classe de l'école élémentaire sauf le CP. Ces manuels, respectivement de CE1 (publié en 2014), de CE2 (publié en 
2011), de CM1 (publié en 2012) et, enfin, de CM2 (publié en 2013), nous paraissent exemplaires pour ce qui touche aux repères de progression concernant la phrase.

Le tableau ci-dessous présente les titres des chapitres dans la partie intitulée «Phrase ». Ces titres sont révélateurs des savoirs visés.

\begin{tabular}{|c|c|c|c|}
\hline \multicolumn{4}{|c|}{ Titres des chapitres de la partie « Phrase » } \\
\hline CE1 & CE2 & CM1 & CM2 \\
\hline $\begin{array}{l}\text { - Je reconnais la } \\
\text { phrase }\end{array}$ & $\begin{array}{l}\text { - Identifier une } \\
\text { phrase }\end{array}$ & $\begin{array}{l}\text { - Identifier la phrase } \\
\text { interrogative }\end{array}$ & $\begin{array}{c}\text { - Utiliser les types et formes de } \\
\text { phrase }\end{array}$ \\
\hline $\begin{array}{c}\text { - Je reconnais la } \\
\text { phrase } \\
\text { interrogative }\end{array}$ & $\begin{array}{c}\text { - Différencier la } \\
\text { phrase déclarative et } \\
\text { interrogative }\end{array}$ & $\begin{array}{l}\text { - Identifier la phrase } \\
\text { injonctive }\end{array}$ & $\begin{array}{c}\text { - Identifier la phrase } \\
\text { exclamative }\end{array}$ \\
\hline \multirow{2}{*}{$\begin{array}{c}\text { - J'utilise la phrase } \\
\text { affirmative et } \\
\text { négative }\end{array}$} & $\begin{array}{c}\text { - Distinguer la forme } \\
\text { affirmative et la } \\
\text { forme négative }\end{array}$ & $\begin{array}{c}\text { - Distinguer la forme } \\
\text { affirmative et la forme } \\
\text { négative }\end{array}$ & $\begin{array}{l}\text { - Identifier les propositions } \\
\text { dans une phrase complexe }\end{array}$ \\
\hline & $\begin{array}{l}\text { - Utiliser la } \\
\text { ponctuation }\end{array}$ & - Utiliser la ponctuation & $\begin{array}{l}\text { - Identifier la proposition } \\
\text { subordonnée relative }\end{array}$ \\
\hline
\end{tabular}

Tableau 1 : La notion de phrase : repères de progression dans les manuels CE1, CE2, CM1 et CM2 de la collection Pépites (Magnard)

Dans ce tableau, plusieurs «facettes » de la notion de phrase sont abordées : la définition de la phrase, ses types et ses formes, les emplois des signes de ponctuation et, enfin, la phrase complexe et ses propositions.

Plusieurs indices montrent que les auteurs du manuel Pépites se sont interrogés sur la progression des notions. Tout d'abord, la définition de la phrase mettant à l'honneur les critères sémantique et typographique ( « Je sais qu'une phrase est un ensemble de mots qui a un sens. Une phrase commence par une majuscule et se termine par un point. Les étoiles brillent dans le ciel. » (Pépites CE1, 2014 : 119)) n'est explicitement présente que dans le manuel de CE1, ce qui tend à montrer l'inutilité de répéter cette définition à tous les niveaux d'enseignement. Les notions de types et formes de phrase sont en outre envisagées avec une précision croissante du CE1 au CM1 avant le chapitre-bilan du manuel CM2 nommé «Utiliser les types et formes de phrase ».

Le choix des verbes dans les titres de chapitre («reconnaitre, différencier, identifier, distinguer ») révèle que la posture métalinguistique est privilégiée dès le CE2 par les auteurs, lesquels reprennent les termes employés par les programmes de 2008. Le savoir-écrire semble plus travaillé dans les chapitres commençant par le verbe «utiliser » : en CE2 et CM1, il s'agit de «savoir utiliser la ponctuation » et, en CM2, de «savoir utiliser les types et formes de phrase». Toutefois, le transfert des savoirs plutôt déclaratifs des manuels est loin d'être automatique chez les élèves lors d'une tâche d'écriture. Comme les programmes le reflétaient déjà, la notion de phrase est surtout abordée dans une perspective de réception 
et non de production dans cette sorte d'ouvrages didactiques. Qu'en est-il des compétences procédurales des élèves en situation d'écriture ? C'est à cette question que notre étude s'est attachée.

\section{Segmentation en phrases et progressivité des apprentissages : éléments de méthodologie}

Nous allons présenter le corpus étudié, recueilli sur une année scolaire (des études ultérieures pourront se prêter à une approche plus longitudinale).

\subsection{Le corpus de l'étude}

Nous travaillons, dans le cadre de cet article, sur des extraits du corpus de la thèse de Véronique Larrivé ${ }^{5}$ (2014), textes recueillis dans des classes de CM2 et de $6^{\text {ème }}$. Nous avons été intéressées par la nature des écrits collectés, qui relèvent de ce que Larrivé (2014) appelle le «journal de personnage », inspiré des « carnets de lecture » développés en didactique de la littérature. Larrivé (2014 : 369) décrit ainsi les textes produits :

Le journal de personnage est un ensemble de textes fictionnels écrits par un même élève pendant la lecture d'une œuvre littéraire. À chaque épisode majeur du récit, l'élève-lecteur est invité à écrire une page du journal en adoptant le «je » d'un personnage donné de la fiction, avec des consignes d'écriture toujours différentes qui permettent de varier les situations d'énonciation et les types de discours à produire.

Ces écrits s'apparentent à ce que Bucheton \& Chabanne (2002) ont appelé « écrits réflexifs », présentés par Cellier \& Dreyfus (2003) de la manière suivante :

Ces écrits sont avant tout personnels, ils accompagnent la réalisation de la tâche, ils sont produits par les dispositifs d'enseignement et soutiennent l'action individuelle et autonome des élèves.

Les textes de cette sorte, rarement corrigés par l'enseignant, marquent néanmoins une étape du travail réalisé. Aussi, nous pensons que les écrits de travail sont des supports pertinents pour évaluer les apprentissages effectifs de jeunes scripteurs. La prise en compte (ou non) d'un éventuel destinataire à l'écrit peut être mesurée. Or, c'est bien cette prise en compte qui est au cœur des apprentissages rédactionnels et de la réflexion sur la progressivité des apprentissages de l'écrit.

Les classes que nous avons sélectionnées dans le corpus de Larrivé (2014) sont deux classes de CM2 d'une grande ville. Celle que nous désignerons par E est composée de 29 élèves; celle nommée L comprend 21 élèves. Ces deux classes ont suivi l'ingénierie didactique proposée par V. Larrivé. Il s'agissait d'étudier pas à pas l'œuvre L'Épopée de Gilgamesh (Folio Junior, 2009). Tout au long de la lecture, les enseignants, expérimentés dans les deux cas, invitaient les élèves à consigner plusieurs types d'écrits autour des personnages de l'œuvre, tels des textes narratifs, descriptifs, argumentatifs mais aussi des dialogues. Dans cette séquence, dix-sept écrits ont été produits. Les textes examinés s'appuient sur la consigne suivante : «Gilgamesh écrit sur une tablette qu'il intitule "le secret de ma sagesse". Imagine et rédige ce que Gilgamesh écrit sur cette tablette ». Cette même consigne a été proposée aux élèves à deux reprises, et ce à quatre mois d'intervalle : pour la classe E, en octobre (date du premier texte du projet) et en février (où a été remis le dernier texte du projet, soit le dix-septième) de l'année 2012 ; pour la classe 
L, en novembre et en mars de cette même année. L'écart temporel permet d'interroger la progressivité des apprentissages pour un élève donné.

Nous avons retenu les productions de quatre élèves (dont les textes intégraux des premiers jets, avec orthographe et mise en page d'origine, ont été joints dans les annexes), ce qui permet une approche qualitative fine et précise des indicateurs de progressivité concernant la segmentation en phrases des textes. Notre objectif est de comparer, non pas les classes, mais, d'une part, les textes d'un même élève et, d'autre part, les textes d'une même classe.

Les classes de $6^{\text {ème }}$ de notre corpus ont été écartées. En effet, même si celles-ci suivaient le même scénario que les classes de l'école, la séquence du collège a été mise en œuvre dans un délai beaucoup plus resserré (de deux semaines). Nous ne disposions donc plus de cet écart dans le temps, condition nécessaire pour mesurer la progressivité des acquis pour les élèves observés.

\subsection{La phrase « graphique » vs la phrase « syntaxique »}

Nous avons choisi de segmenter les textes d'élèves en nous appuyant sur une phrase définie à partir d'un critère d'une part unique (garantissant de ce fait une homogénéité) et d'autre part relationnel, eu égard à son rôle de cadre d'analyse en syntaxe (voir aussi Paolacci \& Rossi-Gensane, 2012 ; Paolacci \& RossiGensane, 2014). Cette phrase dénommée «syntaxique » correspond à « une structure constituée d'un [pivot ${ }^{6}$ ] et de l'ensemble des rapports de dépendance contractés autour de ce [pivot], directement [...] ou indirectement» (Feuillard, 1989: 214). Le pivot est « un noyau relationnel et non sémantique, ce qui signifie [qu'il] n'est pas forcément l'élément le plus important du point de vue du sens, mais celui auquel se rattachent les divers constituants d'une structure [...]» (Feuillard, 1989 : 65). Par exemple, Cet élève adore la grammaire mais il déteste les mathématiques. se décompose en deux phrases «syntaxiques » comportant les pivots respectifs adore et déteste. Formidable, la grammaire! correspond à une seule phrase «syntaxique», organisée autour du pivot, cette fois-ci non verbal, formidable. La phrase «syntaxique » permet une dissociation entre la segmentation elle-même et les effets de la segmentation présents sous la forme de signes de ponctuation et/ou de connecteurs, mais aussi parfois absents. En mesure de segmenter des productions écrites non normées, la phrase «syntaxique » semble ainsi susceptible de rendre compte des compétences effectives - intermédiaires - des élèves ${ }^{7}$. Précisons que le recours à la phrase «syntaxique » peut être complété, sur un autre plan, par la prise en considération de la phrase «graphique » ((uniquement) signalée par une ponctuation forte), qu'il convient, en effet, de ne pas « exclure du langage objet » (Berrendonner \& Reichler-Béguelin, 1989 : 124). On notera à cet égard que l'exemple supra, Cet élève adore la grammaire mais il déteste les mathématiques., s'il contient deux phrases «syntaxiques », consiste en une seule phrase « graphique $»^{8}$.

\subsection{Vers une cartographie des apprentissages rédactionnels}

Comment viser une cartographie des apprentissages rédactionnels concernant le découpage en phrases ? Le tableau ci-dessous présente des indicateurs permettant d'aborder les caractéristiques démarcatives des textes de façon critériée. La flèche qui recouvre les différentes «étapes » de progressivité symbolise une dynamique des apprentissages dans laquelle l'enseignant s'efforce d'inscrire les élèves. On peut imaginer que le curseur se déplace sur l'axe, matérialisant ainsi les acquis rédactionnels, certains indicateurs (dans les colonnes de droite du tableau) étant appréciés plus positivement que d'autres, comme il sera expliqué ci-après. 


\begin{tabular}{|c|c|c|c|c|}
\hline & $\mathbf{A}$ & B & $\mathbf{C}$ & D \\
\hline $\begin{array}{c}\text { Rapport des } \\
\text { phrases } \\
\text { « graphiques » et } \\
\text { des phrases } \\
\text { « syntaxiques » }\end{array}$ & $\begin{array}{c}\text { Phrases } \\
\text { « graphiques » } \\
\text { recouvrant } \\
\text { généralement } \\
\text { plusieurs phrases } \\
\text { « syntaxiques » (ou } \\
\text { nombre de phrases } \\
\text { « graphiques » très } \\
\text { inférieur au nombre } \\
\text { de phrases } \\
\text { «syntaxiques ») }\end{array}$ & $\begin{array}{c}\text { Phrases } \\
\text { « graphiques » } \\
\text { recouvrant } \\
\text { souvent plusieurs } \\
\text { phrases } \\
\text { « syntaxiques » } \\
\text { (ou nombre de } \\
\text { phrases } \\
\text { «graphiques » } \\
\text { inférieur au } \\
\text { nombre de } \\
\text { phrases } \\
\text { « syntaxiques ») }\end{array}$ & $\begin{array}{l}\quad \text { Phrase } \\
\text { «graphique » } \\
\text { allant vers une } \\
\text { coextensivité à } \\
\text { la phrase } \\
\text { « syntaxique» }\end{array}$ & $\begin{array}{c}\text { Phrase } \\
\text { «graphique » } \\
\text { souvent } \\
\text { coextensive à la } \\
\text { phrase } \\
\text { « syntaxique » }\end{array}$ \\
\hline $\begin{array}{l}\text { Emploi des bornes } \\
\text { (matérialisation ou } \\
\text { non des bornes) } \\
\text { entre les phrases } \\
\text { « syntaxiques » }\end{array}$ & $\begin{array}{l}\text { De zéro borne à un } \\
\text { début de } \\
\text { matérialisation des } \\
\text { bornes }\end{array}$ & $\begin{array}{c}\text { Matérialisation } \\
\text { inégale des bornes }\end{array}$ & $\begin{array}{c}\text { Vers une } \\
\text { normalisation } \\
\text { des bornes }\end{array}$ & $\begin{array}{l}\text { Matérialisation } \\
\text { systématique } \\
\text { des bornes }\end{array}$ \\
\hline $\begin{array}{c}\text { Nature des bornes } \\
\text { des phrases } \\
\text { « syntaxiques » }\end{array}$ & $\begin{array}{l}\text { Des connecteurs du } \\
\text { type et, alors, mais à } \\
\text { un début de recours à } \\
\text { la ponctuation forte }\end{array}$ & $\begin{array}{l}\text { Enrichissement } \\
\text { des connecteurs, } \\
\text { ponctuation forte }\end{array}$ & $\begin{array}{c}\text { Présence de } \\
\text { cadratifs (tel, } \\
\text { par exemple, un } \\
\text { jour, suivant le } \\
\text { type de texte } \\
\text { produit), } \\
\text { ponctuation } \\
\text { forte stabilisée }\end{array}$ & $\begin{array}{c}\text { Emploi } \\
\text { diversifié de la } \\
\text { ponctuation et } \\
\text { des connecteurs, } \\
\text { enrichissement } \\
\text { des cadratifs }\end{array}$ \\
\hline
\end{tabular}

Tableau 2 : Indicateurs de progressivité des apprentissages pour le découpage des textes en phrases

Selon les textes, les phrases «syntaxiques» sont, ou non, plus nombreuses que les phrases «graphiques ». Dans le cas, particulièrement répandu dans les textes de scripteurs débutants, où le nombre de phrases «syntaxiques » dépasse celui de phrases "graphiques », ces dernières tendent vers une certaine longueur, tandis que les textes d'élèves plus avancés présentent davantage un équilibre entre phrases «syntaxiques» et phrases «graphiques", de nombre alors approchant et plus souvent coextensives. En effet, comme le montre Chervel (2008), dès la fin du XIX ${ }^{\text {ème }}$ siècle, les phrases «graphiques » longues sont dévalorisées dans les rédactions d'école primaire. Ainsi, Carré \& Moy (1884 : 22), cités par Chervel (2008 : 711), émettent le conseil suivant : «Lorsqu'on s'aperçoit que l'on a fait une phrase longue, on doit la couper en plusieurs phrases ». 
La longueur des phrases «graphiques » peut tout d'abord être due à l'absence de signes de ponctuation ou, tout au moins, à leur rareté, ce que Béguelin (2000) décrit comme un phénomène de sous-ponctuation. Dans un premier cas de figure, non normé, les bornes entre phrases «syntaxiques » ne sont pas matérialisées ou le sont peu, ou encore revêtent une forme particulière, tels les sauts de ligne. Dans un deuxième cas de figure, plus normé, la longueur des phrases «graphiques » est imputable au recours, en tant que bornes, à des connecteurs ${ }^{9}$. Ces derniers, dès lors qu'ils sont surabondants, fonctionnent comme des ponctuants au détriment des signes de ponctuation et sont vus comme autant de traces d'oralité témoignant d'une difficulté de passage à l'écrit ${ }^{10}$, auquel, pour leur part, les signes de ponctuation appartiennent spécifiquement. Le manque, ou non, de diversité des connecteurs fournit également des indications quant au passage à l'écrit, en relation à des stades d'acquisition définis par des psycholinguistes (par exemple, Fayol, 1997, ou Bronckart \& Schneuwly, 1984). Ainsi, dans des textes d'enfants de sept ans, le et, parfois qualifié d'《archiconnecteur», joue le rôle de «prototype de relateurs ». A huit ans, les enfants emploient un paradigme des connecteurs élargi : le et est notamment complété par après, puis, ensuite. Chez les enfants de neuf à douze ans, les connecteurs sont utilisés de manière plus diversifiée au regard de la complexification croissante des écrits produits. Au collège et à l'âge adulte, enfin, l'emploi des connecteurs est soumis à d'importantes variations interindividuelles dépendantes du genre textuel.

À côté des connecteurs, un rôle de segmentation ${ }^{11}$ peut également être accordé aux cadratifs, qui recouvrent notamment les circonstants spatio-temporels en position détachée frontale « à même d'indexer une ou plusieurs propositions » (Charolles \& Péry-Woodley, $2005: 4$ ) à la suite de celle dans laquelle ils se trouvent. Toutefois, alors que « les connecteurs établissent des liens en amont», les «cadratifs, tout en entretenant très souvent des liens avec des segments précédents, sont fondamentalement orientés vers l'aval du discours » (Charolles \& Péry-Woodley, 2005 : 6). Au-delà de leur rôle de segmentation, cette différence dans la directionnalité amène, sur un plan didactique, à opposer les connecteurs, dont la surabondance dans les écrits d'élèves peut révéler une planification «pas à pas » (Schneuwly, 1988), et les cadratifs, pour lesquels « on peut penser que [leur] utilisation [...] est un signe de maitrise de la bidimensionnalité de l'écrit »(Garcia-Debanc, 2010 : 85), dans la mesure où elle s'inscrit dans une visée prospective.

La longueur des phrases « graphiques » peut, enfin, être due à la relative rareté des signes de ponctuation forte et, notamment, au recours à des virgules pour séparer des phrases «syntaxiques ». Cette dernière caractéristique peut être appréciée positivement si l'on considère que l'utilisation de la virgule marque une exploitation (ou un début d'exploitation) du paradigme des signes de ponctuation (par exemple, points de suspension, point d'exclamation, point d'interrogation, ou encore point-virgule), dont la virgule est l'un des plus complexes (voir Catach, $1994: 64-71)^{12}$. Garcia-Debanc (2010:91) remarque à cet égard que, dans son corpus composé de textes de CE2, $6^{\text {ème }}$ et $3^{\text {ème }}$, les virgules «n'apparaissent véritablement que dans les textes de $6^{\text {ème }}$ ». Néanmoins, dans une perspective où la brièveté de la phrase («graphique ») est valorisée, cette utilisation «interphrastique » de la virgule peut aussi être considérée comme relevant d'une compétence intermédiaire. De manière convergente avec cette dernière constatation, Favart \& Passerault (2000) notent, dans une tâche de récit en images impliquant des élèves de CM2, un recours à la virgule à un niveau microstructural du texte et au point à un niveau plus macrostructural (par exemple pour clore ce qui peut être vu comme une séquence textuelle).

Il convient enfin de préciser que certains indicateurs, telle la longueur des textes, qui certes renseignent sur les compétences rédactionnelles des élèves mais ne concernent pas la démarcation, n'ont pas été pris 
en compte. Nous allons maintenant présenter l'analyse de nos résultats en nous attachant à des cas contrastés.

\section{La segmentation en phrases : quelle progressivité au cours d'une année ?}

Le corpus sélectionné présente des compétences rédactionnelles confortant l'idée que l'hétérogénéité des élèves d'une même classe - aspect didactiquement difficile à prendre en compte par l'enseignant - est une réalité. Néanmoins, deux textes de consigne identique à quatre mois d'intervalle sont révélateurs des progrès (ou de l'absence de progrès) des élèves.

Dans le cadre de cet article seront explorés les écrits de quatre élèves que nous trouvons représentatifs du corpus étudié. Nous parlons ainsi de «portraits»d'élèves. Antonio et Carolina sont des élèves dits «en difficulté » à tous les niveaux impliqués dans la production d'un texte (orthographe, syntaxe, cohésion, etc.). Romain comme Nathan sont des élèves dits « sans difficulté » ${ }^{13}$.

\subsection{Les textes d'Antonio et de Romain : des progrès notables au niveau de la segmentation}

Le premier texte d'Antonio ne présente ni signe de ponctuation (on peut noter toutefois la majuscule à l'incipit du texte), ni connecteur. C'est un texte «écrit au kilomètre », sans point final. Les phrases «syntaxiques » se succèdent. Ce texte pourrait être classé dans la colonne A du tableau des indicateurs de progressivité (voir supra). Le texte 2 du mois de mars est beaucoup plus développé. S'il est coextensif à une phrase «graphique » dans la mesure où il comporte un seul point, final, un usage des signes de ponctuation du dialogue s'avère être un critère de progressivité pour cet élève. Le connecteur et (quatre occurrences) participe à la structuration du texte, tout comme le point final, absent dans le texte 1 produit en novembre. Enfin, on peut remarquer, même si elles ne relèvent pas de la démarcation en phrases, les cinq virgules présentes à l'intérieur de la première phrase «syntaxique » qui prouvent également une prise en compte du lecteur. En conséquence, ce deuxième texte peut être classé dans la colonne B du tableau d'indicateurs.

Des éléments de progressivité sont également notables dans les deux productions de Romain. Dans le texte 1 de cet élève, deux phrases « graphiques » correspondent à cinq phrases «syntaxiques ». Outre les deux points, les frontières entre phrases «syntaxiques » sont systématiquement matérialisées, une fois par une virgule et deux fois par le connecteur et. À l'intérieur des phrases « syntaxiques », la répétition de que (précédé à deux reprises par une virgule dans la deuxième phrase «syntaxique ») structure le texte. Que, subordonnant polyvalent, ouvre des subordonnées variées (circonstancielles de conséquence, relatives), même si certaines sont non standard, telles les trois relatives décumulées de la deuxième phrase « syntaxique », où la fonction sujet est assumée, non par un pronom relatif, mais par le pronom personnel je (je suis un homme que je n'ai peur de rien, que je serai peut-être un dieu, que je refferai tous les pays qui croierai). Ce premier texte pourrait être classé dans la colonne B du tableau d'indicateurs. Le texte 2 de Romain, découpé en trois paragraphes, présente, bien que deux bornes n'aient pas été matérialisées (la seconde borne manquante découle du rattachement de maintenant à la phrase «syntaxique » qui suit, dernière phrase «syntaxique » du texte), une segmentation plus spécifique de l'écrit. Ainsi, même s'il ne s'agit pas de démarcation en phrases, il est important de souligner que trois virgules internes à la phrase «syntaxique » sont présentes dans ce texte du mois de mars, comme dans l'exemple suivant où la virgule clôt le circonstant frontal: Grace au parcours que j'ai fait, j'ai apris des choses. Dans le premier 
paragraphe, le segment (et) (aussi) grace a [sic], introduisant ce qui peut être vu comme un complément circonstanciel de manière, participe à la structuration du texte 2 au début de quatre phrases «graphiques ». Hors la première occurrence, il convient néanmoins de noter que, nonobstant la ponctuation forte, le complément circonstanciel de manière est incident à ce qui précède (et ce, malgré, pour la deuxième occurrence, la présence à droite d'une virgule). On a alors affaire à des « compléments différés » (Blanche-Benveniste, 1997 : 114), où, cas de figure beaucoup plus rare, la phrase « graphique » est incluse dans la phrase «syntaxique ${ }^{14}$. En dépit de ce qui, chez un jeune scripteur, sera interprété comme un phénomène de sur-ponctuation, le texte 2 illustre bien, par ses diverses modalités de découpage du texte (ponctuation forte, connecteurs, paragraphes), la prise en compte du lecteur et pourrait être classé dans la colonne $\mathrm{C}$ du tableau d'indicateurs. Hors les «compléments différés », à part, on remarque d'ailleurs dans ce deuxième texte, à la différence du premier, un cas de congruence entre phrase «graphique » et phrase «syntaxique », allant, en cohérence avec la préconisation de brièveté rappelée par Chervel (2008), dans le sens d'un resserrement des phrases (Endiku ma rappeler comment j'étais si méchant quand il n'était pas là.).

\subsection{Les textes de Carolina et de Nathan : des progrès à la marge au niveau de la segmentation}

Les indices de progressivité des textes de Carolina et de Nathan, au niveau de la segmentation en phrases, sont plus ténus que ceux des textes d'Antonio et de Romain.

Dans le texte 1 de Carolina, on note, en relation à la présence de trois points, trois phrases « graphiques ». On peut souligner la présence, à quatre reprises, du connecteur et (orthographié « est » trois fois), deux absences de bornes entre phrases «syntaxiques » ainsi que, de manière non normée, l'apparition d'un que non pas subordonnant mais connecteur (voir Groupe de Fribourg, 2012 : 278, et Deulofeu, 1999, cité par Groupe de Fribourg, ibid.). Ce texte 1 est marqué par des répétitions lexicales et comporte, sur un plan syntaxique, à la fois des détachements et des tours à présentatif avec ou sans clivage. L'extrait suivant (où, également, la deuxième phrase «syntaxique » débute par le que connecteur mentionné supra) illustre ces caractéristiques : j'aurais bien aimé refaire tout ses voyage manifique // que j'ai apprie que la sagesse cettait une chosse manifique d'être gentil avec tu le monde // est sais en particulier avec une personne très gentil qui m'a apprie comment c'ettait bien dêtre gentil avec tu le monde // est c'est cette perssonne. // Dans le texte 2 de février, une frontière entre phrases «syntaxiques » n'est pas matérialisée et, en l'absence de point final (seconde borne manquante), il n'apparaît aucune phrase «graphique » délimitée par des signes de ponctuation forte. Cependant, Carolina emploie d'autres signes, tel, deux fois, le deux-points. Nous pouvons également remarquer que deux connecteurs, et et mais, sont présents dans ce texte. En conclusion, les deux textes de Carolina, assez proches au niveau de la segmentation en phrases, seraient classés dans la colonne A du tableau, aucun indicateur de progressivité ne pouvant être mis en exergue pour les productions de cette élève.

Pour sa part, Nathan use à deux reprises de la majuscule après la virgule dans son texte 1, attribuant ainsi une fonction forte à ce signe de ponctuation (bien que la première virgule, qui borne sur la droite depuis fonctionnant ici comme complément circonstanciel de temps, soit interne à la phrase « syntaxique »). Le texte du mois d'octobre sans point final possède quatre phrases «graphiques » pour sept phrases «syntaxiques ». La répétition de je suis à l'incipit de phrases, soit «graphiques », soit « syntaxiques », rythme le début et la toute fin du texte. Outre depuis, on peut remarquer la présence, à l'initiale d'une phrase «syntaxique », de maintenant (précédé du connecteur et), qui fonctionne également comme 
complément circonstanciel de temps. Autrement dit, sont utilisés deux fois des éléments cadratifs ou, tout au moins, candidats à l'être dans la mesure où leur portée semble d'abord circonscrite à leur phrase «syntaxique »d'accueil. Les compétences de segmentation de ce texte 1 pourraient être positionnées dans la colonne $\mathrm{C}$ du tableau d'indicateurs. Dans le texte 2 du mois de février de ce même élève, des divergences d'évolution peuvent être notées quant à certains critères de progressivité. Alors que, à l'exception du point final, les bornes des phrases « syntaxiques » étaient systématiquement matérialisées dans le texte 1, elles sont six fois absentes dans le texte 2 (l'omission du point final persistant). Ainsi, dans l'extrait suivant, les frontières intermédiaires (à l'endroit des première et deuxième doubles barres) n'apparaissent ni sous forme de signe de ponctuation, ni sous forme de connecteur : et ils nous ont puni // ils ont tué Endiku // j'en pleure toujours//. En revanche, dans d'autres passages, les bornes sont constituées de virgules et/ou de connecteurs, caractérisés par une grande diversité, tels et, donc, mais, ensuite. On peut en outre remarquer que l'adverbe ensuite a un rôle ambigu et pourrait tout autant être considéré comme un cadratif, dont la portée, ici, irait au-delà de la phrase «syntaxique »d'accueil. Deux emplois de depuis peuvent aussi être notés avec, dans le second cas, une portée s'étendant à la phrase «syntaxique » subséquente. Il est intéressant, pour cette dernière occurrence, de constater qu'elle est encadrée par des virgules (internes, donc, à la phrase «syntaxique »). En définitive, si le texte comporte des connecteurs diversifiés et des cadratifs, l'absence de ponctuation forte (aucune phrase " graphique », au sens strict, pour dix-huit phrases «syntaxiques ») incite à le classer, tout comme le texte 1, dans la colonne $\mathrm{C}$ (voire dans la colonne $\mathrm{B}$ ) du tableau d'indicateurs. La présence, à l'issue de la première phrase Je suis devenu sage après tous mes périple, d'un deux-points ouvrant sur une énumération de type plutôt argumentatif, amène d'ailleurs à se demander si cette hésitation entre les types textuels n'aurait pas induit dans le texte 2 une désorganisation de la segmentation ${ }^{15}$. Quoi qu'il en soit, de même que pour les productions de Carolina, les indices de progressivité restent particulièrement minces pour les deux productions de cet élève.

\section{Conclusion : pour un enseignement explicite de la segmentation en phrases}

Au terme du travail de description des textes produits, on note que l'écriture régulière - rappelons que les élèves observés ont produit dix-sept textes dans le projet de lecture de l'œuvre Gilgamesh - ne constitue pas l'assurance de progrès, tout au moins dans le domaine de la démarcation, comme le montrent particulièrement les productions de fin de séquence d'enseignement de Carolina et de Nathan. La reprise d'une même consigne ne garantit pas non plus pour tous les élèves (qu'ils soient dits en difficulté ou pas) un travail plus abouti.

S'il est important de proposer des situations d'écriture fréquentes, il semble néanmoins tout aussi important de les compléter par des situations de réécriture. Cette réécriture étayée des textes nous paraît pouvoir participer d'une dynamique démarcative. En effet, rappelons que «le savoir-écrire est un "apprenable" » (Halté, 2003 : 37) ; ajoutons même un « enseignable » : l'enseignant a un rôle à jouer dans ces apprentissages complexes. Quelques pistes didactiques permettant de répondre aux besoins des élèves peuvent être suggérées. Pour la tranche d'âge envisagée (cycle 3), nous croyons, avec d'autres didacticiens, au pouvoir de la lecture à haute voix et de la réception des textes par des pairs. Jaffré (2014 : 133-134) souligne :

La pédagogie de la ponctuation est de ce fait indissociable d'activités capables de donner à un texte une existence sociale. [...] L'oralisation systématique de ce qui est écrit permet de prendre la mesure d'une structure qu'il faut rendre visible et donc lisible. 
L'oralisation révèle ainsi des aspects qui n'avaient pu être traités par de jeunes élèves dans le passage à l'écrit, lors de la mise en mots.

Il est également envisageable de «découper», au sens propre, concret, du terme, le texte en phrases «graphiques », en s'inspirant de l'éloge du «bricolage » grammatical de Sève \& Ambroise (2009). Les élèves prennent alors conscience matériellement de la nécessité de segmenter pour éviter le texte monobloc difficilement lisible (c'est le cas pour le texte 2 d'Antonio). Après cette étape de mise en évidence de la longueur des phrases «graphiques» se pose la question du découpage au sein de ces mêmes phrases : la segmentation anticipée en phrases «syntaxiques » par l'enseignant peut y aider (avec les doubles barres). L'élève voit son travail valorisé dans la mesure où son texte n'est révisé que de façon minimale, comme il est illustré dans cet extrait du texte d'Antonio : et je les remerci mintenan // je vais veiller sur mon peuple // je vais aussi le protéger de tous les danger aunorer encore plus les dieux et surtou Slamash le dieu du soleil qui a aussi protéger Endiku et moi // et je sais qu'il mecoute en ce moment même préci // et je sais aussi qu'il est fier de moi davoir atain la sagesse. // Il s'agit de développer chez les élèves l'idée que la ponctuation n'est pas ornementale, qu'elle possède plusieurs rôles (dont un rôle syntaxique) et qu'elle aide le lecteur à mieux lire/comprendre les textes.

L'enseignant ne doit pas oublier que «l'apprentissage de la ponctuation s'inscrit dans une scène didactique où "le lecteur est l'acteur principal de la pièce" [la formule entre guillemets est de Jaffré (2014 : 133)] » (David \& Vaudrey-Luigi, $2014: 6$ ) et doit viser la prise en compte du lecteur par l'élève. Pour chacun des élèves observés, un aspect particulier favorisant la réécriture des textes produits pourrait être mis en avant par l'enseignant. Par exemple, Romain pourrait retravailler le premier paragraphe de son texte 2 : Grace au parcours que j'ai fait, j'ai apris des choses. Et aussi grace a la mort de Endiku, // sa ma tellement choquer que je suis devenu encore plus sage. // Pour moi c'est un brave guerrier // c'est pur ça que je suis devenu un brave sage. Aussi, grace à ce que m'a apris Utanapichtim se lui qui a la vie eternelle. Et aussi grace aux vilageois qui m'encouragait pour tuer Umbaba le Dieu de la forêt. // Dans ce passage, comme il a déjà été évoqué supra, la répétition de grâce à participe à la multiplication des phrases «graphiques » qui se réduit en définitive à une énumération répondant à la première phrase $j$ 'ai apris des choses grace à... La réécriture de l'extrait pourrait être facilitée par le rapprochement des éléments introduits par grâce à, mis en évidence, par exemple, par une représentation en grille (telle qu'élaborée, aux côtés de C. Blanche-Benveniste, par le cadre aixois de micro et macrosyntaxe (voir, par exemple, Blanche-Benveniste, 1997)).

Enfin, nous pensons que le recours à des grammaires scolaires comme celle de Chartrand \& Simard (2000), qui accorde une place centrale à la copie d'élève, devrait être favorisé. En effet, le chapitre consacré à la ponctuation (chapitre 3 de cette grammaire) se clôt par un travail sur un écrit d'élève à réviser ( «J'utilise mes connaissances pour réviser la ponctuation»). Il est dommage que la plupart des manuels d'aujourd'hui travaillent les notions grammaticales à partir de textes d'auteurs, ce qui apparaît encore comme une façon de minorer la production au profit de la réception.

Nous espérons que cette étude participera à l'établissement d'une cartographie des compétences rédactionnelles des élèves dans le domaine de la démarcation, aidant ainsi les enseignants à mieux répondre aux difficultés perçues dans les productions.

\section{Annexes}

Texte 1 d'Antonio (novembre) de la classe $L$ 
Moi Gilgamesh j'écrit mer aventure sur cette tablette // je vai comencer par la premier aventure // j'ai fais le tour du monde avec mes muscle // j'ai combtu pour me défendre //

\section{Texte 2 d'Antonio (mars) de la classe $\mathbf{L}$}

Le secret de ma sagesse c'est toute les aventures que j'ai vécu avec Endiku, la fille qui la emmener ici j'uska Uruk, Shamash le dieu du soleil, qui éder a combatre Umbaba, le terre séleste, et plin d'autre monstre et aussi se qu'ils ont fait pour moi // Endiku et rester tout le temp avec moi // et même si j'avai une [mot illisible] difficile de par exemple tuer Umbaba il ma suivi sens se dire //«nont // je ni vais pas»// il a toujours dit // « oui // je te suivre » // il ma toujour défendu et Shamash qui nous a protéger dans tout les périls qui mes arriver ${ }^{16} / /$ et je les remerci mintenan // je vais veiller sur mon peuple // je vais aussi le protéger de tous les danger aunorer encore plus les dieux et surtou Shamash le dieu du soleil qui a aussi protéger Endiku et moi // et je sais qu'il mecoute en ce moment même préci // et je sais aussi qu'il est fier de moi davoir atain la sagesse. //

\section{Texte 1 de Romain (novembre) de la classe $\mathbf{L}$}

Je suis redoutable tellement que je vais accomplir des choses que nule personne peut accomplir que j'irai gouverner le monde que tous le monde s'inclinerai devant moi, // je suis un homme que je n'ai peur de rien, que je serai peutêtre un dieu, que je refferai tous les pays qui croierai. // Je turai des bêtes féroces des redoutables rien que pour manger // est je suis très forts que je pourrait mêtre tous les pays en harmoni et que je règnerait sur tous les pays qui me gouverneront comme rois et que je pourrai donner à manger à tous les monde et que préparerait les patrouilles pour si jamais quelqu'un s'attaque // et j'envorait m'est troupe pour aller les tuers. //

\section{Texte 2 de Romain (mars) de la classe $\mathbf{L}$}

Grace au parcours que j'ai fait, j'ai apris des choses. Et aussi grace a la mort de Endiku, // sa ma tellement choquer que je suis devenu encore plus sage. // Pour moi c'est un brave guerrier // c'est pur ça que je suis devenu un brave sage. Aussi, grace à ce que m'a apris Utanapichtim se lui qui a la vie eternelle. Et aussi grace aux vilageois qui m'encouragait pour tuer Umbaba le Dieu de la forêt. //

Endiku ma rappeler comment j'étais si méchant quand il n'était pas là. // Et tout c'est gents la mon fait comprendre le secret de ma sagesse // et aussi grace à l'amie que j'ai renconter la première fois de la vie ${ }^{17}$. // Je suis convaincu qu'il mon aidé à trouver ma sagesse // et tous ses gents je les protègerais tous du mal. //

Ils mont volé la moitié de mon cœur en tuant Endiku // maintenant, je ne veux pas con arache la deuxième moitié en tuant tous les autres vilageois. //

\section{Texte 1 de Carolina (octobre) de la classe $\mathbf{E}$}

bonjour // je suis le roi Gilgamesh. //

Quand je suis allée en voyage j'ai vécu toute une expérense et plein de mystère. // Les paysages donc je suis allée était magnifique // j'aurais bien aimé refaire tout ses voyage manifique // que j'ai apprie que la sagesse cettait une chosse manifique d'être gentil avec tu le monde // est sais en particulier avec une personne très gentil qui m'a apprie comment c'ettait bien détre gentil avec tu le monde // est c'est cette perssonne // un moment quand je savais que j'aitais le maître j'ai commentçait a commendé les autre // et des fois jallais dans la chambre de mais parent // est quand est venue le dieux du ciel Anu c'est a ce moment que j'ai vraiment comprie a quel poind c'etait important. //

\section{Texte 2 de Carolina (février) de la classe $\mathbf{E}$}

Je suis devenue sage quand j'ai rencontré Enkidu : // Enkidu avait rencontré une courtisane qui s'appelle Anu // et Enkidu me lavez presentait : // je suis devenu sage quand j'avais rencontré des personne qui était gentil // et j'ai battu 
des monstre // mais sa n'a pas denportanse // quand j'ai vu mon ami enkidu mort j'ai repenser au chose qui m'avait appris comme être sage //

\section{Texte 1 de Nathan (octobre) de la classe $\mathbf{E}$}

Je m'appelle Gilgamesh, // je suis le fils de Ninsun la Biflesse et du roi Lugabanda. // Je suis roi d'Uruk un très belle ville. // Je suis devenue sage quand tout les habitant d'Uruk mon dit que je ne pensais qu'à moi et que je ne faisait que me venter d'être le plus fort. // Depuis, Mes soldats même les moins braves n'ont plus du tout peur de moi, alors que je suis toujours roi d'Uruk, mi homme-mi divin, // Et maintenant Anu et tous les autres dieu ne regrètent plus de m'avoir hissé sur le trône d'Uruk. // Je suis devenu sage quand tout les gens m'ont dit qu'ils me détestes //

\section{Texte 2 de Nathan (février) de la classe $\mathbf{E}$}

Je suis devenu sage après tous mes périple : // j'ai tué Kumbala le terrible, // j'ai tué le taureau céleste ce qui a énervé les dieux // et ils nous ont puni // ils ont tué Endiku // j'en pleure toujours // ensuite je suis allé voir Utanapishtim, l'homme qui a obtenu l'imortalité // j'ai fais un long voyage // donc il m'a donné une chance // mais je n'y est pas arrivé // il m'a quand même dit qu'une plante pouvait me rendre imortel // donc je suis partis à sa recherche // je l'ai trouvé dans l'eau // ensuite pour reprendre ma respiration je l'ai posé à côté de moi // et un serpent me l'a pris et est rentré dans son trou, // depuis je trouve que j'ai eu de la chance d'être roi et que la malchance peut arriver à tout le monde, // et, depuis, je nourris tous les gens qui ont faim, // je suis gentil avec tout le monde //

\section{Références bibliographiques}

Béguelin, M.-J. (éd.) (2000). De la phrase aux énoncés : grammaire scolaire et descriptions linguistiques. Bruxelles : De Boeck Duculot.

Berrendonner, A. (2002). Les deux syntaxes. In Charolles, M., Le Goffic, P. \& Morel, M.-A. (éds). Y a-t-il une syntaxe au-delà de la phrase? Verbum, XXIV, 1-2, 23-35.

Berrendonner, A. \& Reichler-Béguelin, M.-J. (1989). Décalages : les niveaux de l'analyse linguistique. Langue française, 81, 99-125.

Blanche-Benveniste, C. (1997). Approches de la langue parlée en français. Gap-Paris : Ophrys.

Bronckart, J.-P. \& Schneuwly, B. (1984). La production des organisateurs textuels chez l'enfant. In Moscato, M. \& Piéraut-Le Bonniec, G. (éds). Le langage : construction et actualisation. Rouen: Presses Universitaires de Rouen, 165-178.

BULLETIN OFFICIEL [B.O.] (2008). Hors-série, 3, 19 juin 2008. Horaires et programmes d'enseignement de l'école primaire. Paris : Ministère de l'Éducation nationale et Ministère de l'Enseignement supérieur et de la Recherche.

Catach, N. (1994). La ponctuation. Paris : Presses Universitaires de France. Collection Que sais-je ?

Cellier, M. \& Dreyfus, M. (2003). Place et fonctions des écrits intermédiaires ou réflexifs dans des dispositifs didactiques pour travailler l'oral. Repères, 26-27, 83-103.

Chabanne, J.-C. \& Bucheton, D. (éds) (2002). Ecrire en ZEP. Un autre regard sur les écrits d'élèves. Paris : Delagrave. CRDP de l'Académie de Versailles.

Charolles, M. \& Péry-Woodley, M.-P. (2005). Introduction [au numéro Les adverbiaux cadratifs]. Langue française, $148,3-8$. 
Chartrand, S. \& Simard, C. (2000). Grammaire de base. Québec : Édition du Renouveau Pédagogique.

Chervel, A. (2008). Histoire de l'enseignement du français du XVIIe au XXe siècle. Paris : Retz.

David, J. \& Vaudrey-Luigi, S. (éds) (2014). Enseigner la ponctuation. Le français aujourd'hui, 187.

Favart, M. \& Passerault, J.-M. (2000). Aspects fonctionnels du point et de la virgule dans l'évolution de la planification des récits écrits. Enfance, 2, 187-205.

Fayol, M. (1997). Des idées au texte. Paris : Presses Universitaires de France.

Feuillard, C. (1989). La syntaxe fonctionnelle dans le cadre des théories linguistiques contemporaines. Thèse d'État : Université Paris V.

Garcia-Debanc, C. (2010). Segmentation, connexion et indexation dans des productions écrites d'élèves de 9 à 13 ans de deux genres textuels. Synergies Pays Scandinaves, 5, 81-96.

Groupe de Fribourg (2012). Grammaire de la période. Berne: Peter Lang. Collection Sciences pour la communication.

Halté, J.-F. (2003). Didactique de l'écriture, didactique du français : vers une cohérence configurationnelle. Repères, 26-27, 31-49.

Jaffré, J.-P. (2014). À quoi sert la ponctuation ?. Le français aujourd'hui, 187, 129-137.

Larrivé, V. (2014). Du bon usage du bovarysme en classe de français. Développer l'empathie fictionnelle des élèves pour les aider à lire les récits littéraires : l'exemple du journal de personnage. Thèse : Université Michel de Montaigne-Bordeaux III (HAL : <NNT : 2014BOR30044>. <tel-01155167>).

Nonnon, É. (2010). La notion de progression au cœur des tensions de l'activité d'enseignement. Repères, 41, 5-34.

Paolacci, V. \& Rossi-Gensane, N. (2012). Quelles images de la phrase dans les écrits d'élèves de fin d'école primaire française ? Description linguistique et réponses didactiques aux difficultés des élèves. Actes du $3^{\text {ème }}$ Congrès Mondial de Linguistique Française, 341-359.

Paolacci, V. \& Rossi-Gensane, N. (2014). Ponctuation et écrits d'élèves : une conception différente de la phrase pour enseigner la ponctuation autrement. In David, J. \& Vaudrey-Luigi, S. (éds). Le français aujourd'hui, 187, 115125.

Pépites (CE1, 2014), (CE2, 2011), (CM1, 2012), (CM2, 2013). Manuels de français coordonnés par SavadouxWojciechowski, C. Paris : Magnard.

Riegel, M., Pellat, J.-C. \& Rioul, R. (2009). Grammaire méthodique du français. Paris : Presses Universitaires de France (1 ${ }^{\text {ère }}$ édition : 1994).

Schneuwly, B. (1988). Le langage écrit chez l'enfant. Neuchâtel-Paris : Delachaux \& Niestlé.

Sève, P. \& Ambroise, C. (2009). Images, ciseaux, tirettes... Un exemple de bricolage didactique au CE1 autour des relations nom/verbe. Repères, 39, 103-123.

\footnotetext{
${ }^{1}$ Nous remercions vivement les relecteurs anonymes de leurs commentaires.
} 
${ }^{2}$ L'expression figure dans les projets de programmes soumis aux enseignants en 2015 (site consulté le 22 /11/2015 : http://www.education.gouv.fr/cid93042/projet-de-programmes-pour-les-cycles-2-3-et-4.html).

${ }^{3}$ Nous entendons par «facettes » un «élément de connaissance » (Tiberghien \& Malkoun, 2007, cités par Nonnon, 2010 : 8). Une notion grammaticale est ainsi constituée de plusieurs « facettes ».

${ }^{4}$ On peut noter que l'exclamation est mise sur le même plan que les trois premières modalités, bien qu'elle se cumule avec chacune d'entre elles, alors que celles-ci sont mutuellement exclusives.

${ }^{5}$ Nous remercions vivement Véronique Larrivé (professeur à l'ESPE de l'Académie de Toulouse) de nous avoir permis de travailler sur le corpus de sa thèse.

${ }^{6}$ Nous nous sommes permis de remplacer le terme « prédicat », polysémique, par celui de « pivot ».

${ }^{7}$ On se reportera aux textes d'élèves indiqués dans les annexes, où les doubles barres (//) marquent le découpage en phrases dites « syntaxiques ».

${ }^{8}$ En revanche, Cet élève adore la grammaire mais déteste les mathématiques. serait analysé comme une seule phrase « syntaxique » organisée autour de deux pivots considérés comme coordonnés suite à la mise en commun du sujet.

${ }^{9}$ Il convient de préciser que, dans cet article, le terme de connecteur est réservé aux éléments nommés, par exemple par Riegel et al. (2009), « conjonctions de coordination» et «adverbes conjonctifs », à la condition toutefois qu'ils relient des phrases «syntaxiques » (et non d'autres éléments de même rôle ou même fonction syntaxique : dans ce dernier cas, ils seraient considérés comme des coordonnants).

${ }^{10}$ Notons, sans que ce point puisse être développé ici, que les connecteurs, certes communs à la langue écrite et à la langue orale, y sont cependant employés différemment.

${ }^{11}$ Signalons néanmoins que, de façon normée, les cadratifs à l'intérieur du texte doivent être précédés d'un signe de ponctuation et/ou d'un connecteur (il s'agit souvent d'un signe de ponctuation forte).

${ }^{12}$ Toutefois, cette complexité concerne avant tout l'emploi de la virgule à l'intérieur des phrases « syntaxiques », qui sera peu évoqué dans cette étude portant sur la démarcation entre phrases.

${ }^{13}$ Nous reprenons les prénoms énoncés dans la thèse de Véronique Larrivé pour que les lecteurs puisssent y retrouver les références. Rappelons que, dans les textes, les doubles barres (//) marquent le découpage en phrases dites «syntaxiques ».

${ }^{14}$ Si l'on privilégie une segmentation de « rattachement». Une autre possibilité de segmentation consisterait à traiter ces « compléments différés » comme autant de phrases «syntaxiques ». Pour les troisième et quatrième occurrences introduites par (et) (aussi) grace a/aux, il y aurait alors coïncidence entre phrase «graphique» et phrase «syntaxique ».

${ }^{15}$ Comme l'a suggéré l'un des relecteurs anonymes.

${ }^{16}$ Remarquons ici que le rattachement, de manière normée, de et Shamash qui nous a protéger dans tout les périls qui mes arriver à il ma toujour défendu nécessite le remplacement de et par ainsi que ou par comme.

${ }^{17}$ Le segment et aussi grace à l'amie que j'ai renconter la première fois de la vie, ne pouvant dans ce cas être rattaché à la phrase «syntaxique » précédente, doit lui-même être considéré comme une phrase «syntaxique ». 